\title{
Hauptstadtkongress Medizin und Gesundheit 2017
}

\section{Die Pflege im Dialog mit Politik, Gesundheitswirtschaft und Verbänden}

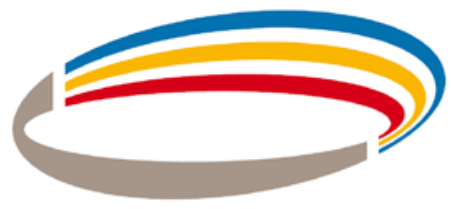

HAUPTSTADT KONGRESS 2017

" Der Hauptstadtkongress Medizin und Gesundheit ist die Leitveranstaltung der Gesundheitsbranche. Vom 20. bis 22. Juli 2017 debattieren 8.000 Entscheidungsträger aus Krankenhäusern und Kliniken, aus Ärzteschaft und Pflege, aus der Gesundheitspolitik und vonseiten der Kostenträger eine Vielzahl kontroverser Fragen des Gesundheitswesens.

Der Deutsche Pflegekongress ist einer von drei Fachkongressen, die unter dem Dach des Hauptstadtforums Gesundheitspolitik stattfinden. Der Pflegekongress widmet sich in diesem Jahr schwerpunktmäßig dem Thema „Managen und Führen": Vorgestellt werden innovative auf Personalbindung zielende Konzepte und neue Ansätze zur Schaffung besserer Karrieremöglichkeiten. Themen sind auch Fachkräftemangel, Personalakquise, Arbeitgeberqualität und Pflegeberufegesetz. Präsentiert werden dazu auch erfolgreiche Beispiele aus der Praxis.

Den Deutschen Pflegekongress zeichnet vor allem die Vielfalt seiner Themen und die Spannbreite der Veranstaltungen aus: Pflegestärkungsgesetze, Personalbemessung, Pflegekammern, Akademisierung der Pflege, Qualitätsentwicklung und -berichterstattung, Robotik in der Pflege und die neuen Pflegegrade - das alles und mehr wird ebenfalls Thema sein. Und

nicht zuletzt die Gretchenfrage: Was ist gerechte Bezahlung in der Pflege?

Mit Veranstaltungen wie „Karrierewege und Kompetenzgewinnung in der Praxis und in Hochschulen" oder „Unternehmertum in der Pflege - Probleme, Herausforderungen, Lösungen“ intensiviert der Kongress gezielt den Dialog zwischen Hochschulen, der Praxis der Pflege und den wirtschaftlichen Stakeholdern.

Teilnehmern bietet sich zudem die einmalige Möglichkeit, mit allen Partnern und Akteuren im Gesundheitswesen den fach- und disziplinübergreifenden Dialog aufzunehmen und sich mit Ärzten, Krankenhausmanagern und Politikern auf Augenhöhe auszutauschen.

www.hauptstadtkongress.de www.hauptstadtkongress.de/deutscher-pflegekongress

\section{WHS Startup Track}

\section{Plädoyer für Gründung der Bundespflegekammer}

» Dr. Markus Mai, Präsident der rheinland-pfälzischen Landespflegekammer, hat sich anlässlich des Deutschen Pflegetags 2017 vehement für die Errichtung einer Bundespflegekammer ausgesprochen. Gemeinsam mit anderen Akteuren der Pflege, wie Andreas Westerfellhaus, Präsident des Deutschen Pflegerats, fordert Mai eine institutionalisierte Interessenvertretung für die Pflegenden auch auf Bundesebene. „Neben der klaren Positionierung in den einzelnen Bundesländern müssen auch zwingend der Bundesgesetzgeber und die Bundesregierung von uns Pflegenden direkt über unsere Forderungen informiert werden. Gerade auf Bundesebene werden viele Weichen für die Pflege, aber auch für das gesamte Gesundheitssystem, gestellt“.

Die Bundespflegekammer werde als Spitzenorganisation der pflegerischen Selbstverwaltung auf Bundesebene das berufliche Zusammengehörigkeitsgefühl der professionell Pflegenden stärken, fachliche und berufspolitische Aktivitäten länderübergreifend strategisch ausrichten und die pflegerische Versorgung der Gesellschaft auf nationaler Ebene gestalten. „Wir müssen auch auf Bundesebene dafür sorgen, dass eine sachgerechte und professionelle Pflege der Bevölkerung sichergestellt ist", so Mai.

Für den Gründungsprozess der Bundespflegekammer (in Gründung) soll nach Ansicht der Vertreterversammlung der Kammer in Rheinland-Pfalz eine „Gründungskonferenz zur Errichtung der Bundespflegekammer" mit mindestens den Vertretern der bereits vollständig etablierten Kammern (d.h. in Bundesländern, in denen es bereits Gesetze zur Errichtung von Landespflegekammern gibt) gebildet werden.

Die Bundespflegekammer soll im Laufe der nächsten Legislaturperiode des Bundestages (2017-2021) abschließend gegründet werden und ihre Funktion aufnehmen. Dies wäre ein entscheidendes Signal an die Bundespolitik, heißt es.

\section{www.bundespflegekammer.de}

\section{Bewerbungsphase gestartet}

॥ Vom 15.-17. Oktober 2017 geht es auf dem diesjährigen World Health Summit wieder um die Zukunft der Gesundheitsversorgung und um die neuesten Ideen, Techniken und Therapien. Im Rahmen des WHS Startup Track können innovative Gesundheits-Startups aus aller Welt ihre innovativen Konzepte vorstellen. Die Bewerbungsfrist läuft bis zum 30. Juni 2017.

Insgesamt 25 Bewerber werden zum WHS 2017 nach Berlin eingeladen. Die zehn Finalisten präsentieren am 16. Oktober 2017 ihre Ideen und Konzepte in einem Pitch vor der Jury und den internationalen WHS Teilnehmern aus Wissenschaft, Wirtschaft, Politik und Zivilgesellschaft. Schirmherr des WHS Startup Track ist Bundesgesundheitsminister Hermann Gröhe, der die Sieger küren wird. Die Veranstaltung gilt mit mehr als 1.600 Teilnehmern als das wichtigste strategische Forum für weltweite Gesundheitsfragen. 2017/startup-track 